\title{
Epidermal Growth Factor Receptor (ErbBI) Expression in Prostate Cancer Progression: Correlation With Androgen Independence
}

\author{
Rajal B. Shah, ${ }^{1,3}$ Debashis Ghosh, ${ }^{2}$ and James T. Elder ${ }^{4,5,6 *}$ \\ 'Department of Pathology, University of Michigan Medical School, Ann Arbor, Michigan \\ ${ }^{2}$ Department of Biostatistics, University of Michigan School of Public Health, Ann Arbor, Michigan \\ ${ }^{3}$ Department of Urology, University of Michigan Medical School, Ann Arbor, Michigan \\ ${ }^{4}$ Department of Dermatology, University of Michigan Medical School, Ann Arbor, Michigan \\ ${ }^{5}$ Department of Radiation Oncology, University of Michigan Medical School, Ann Arbor, Michigan \\ ${ }^{6}$ Ann Arbor Veterans Affairs Hospital, Ann Arbor, Michigan
}

\begin{abstract}
BACKGROUND. The role of the epidermal growth factor receptor (ErbB1) in the progression of prostate cancer is incompletely understood.

METHODS. Tissue microarrays from hormone-naive and advanced androgen-independent tumors were used to investigate the role of ErbB1 in prostate cancer progression.

RESULTS. ErbB1 expression in tumor tissues was strongly associated with hormonerefractory status (odds ratio $=6.67,95 \% \mathrm{CI}=(2.6,17.4), P=0.0001$ ). However, ErbB1 overexpression was not a statistically significant covariate in a multivariate proportional hazards model for biochemical failure of hormone-naïve prostate cancer. Moreover, ErbB1 overexpression was not associated with tumor differentiation $(P=0.44)$, positive margins $(P=0.53)$, seminal vesicle invasion $(P=0.69)$, extraprostatic extension $(P=0.10)$, or preoperative PSA $(P=0.18)$ in the hormone-naïve group.

CONCLUSIONS. These findings are consistent with a model in which ErbB1 expression increases during the development of the androgen-independent state, and suggest that drugs targeted toward ErbB signaling could be of therapeutic relevance in the management of advanced prostatic carcinoma. Prostate 66: 1437-1444, 2006. (C) 2006 Wiley-Liss, Inc.
\end{abstract}

KEY WORDS: $\quad$ epidermal growth factor receptor; prostate cancer; androgen independence; tumor progression

\section{INTRODUCTION}

Once prostate cancer progresses to a state of androgen independence, therapeutic options are limited and prognosis is invariably poor [1]. The molecular mechanisms for the development of androgen resistance are complex and largely unknown, but likely involve peptide growth factors and their receptors [2]. The majority of androgen-independent prostate cancers (AIPCs) express androgen receptors (AR), suggesting that AR signaling pathways are activated in the absence of androgen. In this respect, a functional crosstalk between growth factors and receptors of the c-ErbB family and AR-activated pathways has been shown in
Abbreviations: AIPC, androgen-independent prostate cancer; AR, androgen receptor; AREG, amphiregulin; EGF, epidermal growth factor; EREG, epiregulin ERK, extracellular signal-responsive kinase; HB-EGF, heparin-binding EGF-like growth factor; RTK, receptor tyrosine kinase; SFK, Src family kinase; TGF- $\alpha$, transforming growth factor- $\alpha$; TMA, tissue microarray.

Grant sponsor: National Cancer Institute; Grant number: P50CA69568; Grant sponsor: Ann Arbor Veterans Affairs Hospital; Grant sponsor: Babcock Memorial Trust.

*Correspondence to: James T. Elder, 3312 CCGC, P.O. Box 0932, University of Michigan, Ann Arbor, MI 48109-0932.

E-mail: jelder@umich.edu

Received 27 January 2006; Accepted 11 April 2006 DOI $10.1002 /$ pros.20460

Published online 1 June 2006 in Wiley InterScience (www.interscience.wiley.com). 
clinical models [3-7]. The mammalian c-ErbB family is comprised of four closely related receptor tyrosine kinases (RTKs) that interact hierarchically in response to multiple ErbB receptor ligands [8,9]. Members of this family include ErbB1, more traditionally known as the epidermal growth factor (EGF) receptor; ErbB2, ErbB3, and ErbB4. Ligand binding to the extracellular domain promotes receptor homo- and heterodimerization, and as a consequence auto- and trans-phosphorylation of specific tyrosine residues on the cytoplasmic domain. These events result in the activation of multiple signal transduction pathways, ultimately affecting many cellular functions including cell migration, proliferation, and differentiation [10].

ErbB receptor tyrosine kinases (RTKs) have been implicated in the progression of a wide variety of human cancers [11,12]. Growth factors of the EGF family, such as EGF itself, transforming growth factor- $\alpha$ (TGF- $\alpha$ ), amphiregulin (AREG), and epiregulin (EREG) heparin-binding EGF-like growth factor (HB-EGF), could contribute to ErbB activation in prostate cancer through autocrine and paracrine mechanisms, resulting in increased proliferation, survival, motility, and invasion of tumor cells. Evidence that such pathways are important in prostate cancer include reports that prostate cancer cells express ErbB family members in vitro and in vivo, and that EGF and TGF- $\alpha$ are potent mitogens for prostatic cancer cells. Moreover, expression of certain ErbB ligands in vivo has been found to correlate with disease progression. Thus, expression of TGF- $\alpha$ was shown to increase with increasing degrees of prostatic malignancy [7], and AREG has been reported to increase during progression from normal epithelium to prostatic intraepithelial neoplasia to prostatic cancer [13].

Previous studies have indicated that ErbB1 is overexpressed in primary and metastatic prostate cancer specimens $[7,14]$. At the time these studies were initiated, no studies had addressed the question of whether expression of ErbB1 is associated with progression to androgen independence. Subsequently, one of two recent studies has suggested that this is the case $[15,16]$. Given the inherent biological variability of prostate cancer and the variety of technical variables that can influence the results, these findings merit further confirmation utilizing samples that have been prospectively collected, fixed, and processed in a uniform way. To this end, we have utilized tissue microarrays (TMAs) to investigate the potential role of ErbB1 in prostate cancer progression by comparing its expression in primary hormone-naïve clinically localized prostate cancer versus advanced metastatic androgenindependent disease.

\section{MATERIALS AND METHODS}

\section{Study Population, Clinical Data, and Prostate Sample Collection}

As a source of hormone-naïve prostate cancers, we utilized tissue microarrays (TMAs) containing 433 cores representing cancer and benign tissue from 112 men who underwent radical retropubic prostatectomy at the University of Michigan as the primary monotherapy (i.e., no adjuvant or neoadjuvant hormonal or radiation therapy) for clinically localized prostate cancer. It was assumed that these tumor samples were hormone-naïve, as these subjects had not yet been subjected to antiandrogen therapy. As a source of AIPC samples, we studied TMAs containing 323 cores ( 1 to 34 per patient) representing all the prostate cancer metastasis sites and the prostate (if present) from 30 patients who died of advanced hormone-refractory prostate cancer at our institution. Patients were identified with hormone-refractory prostate cancer by the Medical Oncology Service of the University of Michigan Comprehensive Cancer Center and rapid autopsies were performed to obtain samples as described previously [17]. Both radical prostatectomy and rapid autopsy series are part of the University of Michigan Prostate Cancer Specialized Program of Research Excellence (S.P.O.R.E.) Tissue Core. All patients provided written informed consent, and this study was approved by the Institutional Review Board of the University of Michigan Medical School. Three cores $(0.6 \mathrm{~mm}$ in diameter) were taken from each representative tissue block to construct the TMAs. The TMA construction protocol has been described $[18,19]$. Detailed clinical, pathological, and TMA data are maintained on a secure relational database as previously described [20].

Within the hormone-naïve group, the median postsurgery follow-up was 53 months (range $=1.9$ 107.6 months) and the average age at surgery was 59.2 years (range $=43-80$ years). Sixty-six percent of tumors were organ-confined (stage pT2), $15.1 \%$ of tumors had signs of local invasion (pT3), and 5.3\% had disease involving the bladder (stage pT4). Among the 112 patients, 28 had biochemical treatment failure defined by a postoperative PSA $>0.2 \mathrm{ng} / \mathrm{ml}$.

Within the hormone-refractory group of 30 men, 28 were initially diagnosed with clinically localized prostate cancer but developed widely disseminated disease after 5-10 years. Two men presented with disseminated disease. The median age at the time of death was 71 years (range $=53-84$ years). Of these 30 patients, 8 men underwent radical prostatectomy, and 17 received external beam radiation as their primary treatment. Twenty-eight men received combination 
therapy, and all underwent hormonal manipulation. Each of the eight men who underwent radical prostatectomy as primary treatment experienced a recurrence, received additional treatment (hormonal manipulation with or without radiation and/or chemotherapy), and then progressed to the hormonerefractory state. The time that patients were treated with androgen deprivation therapy ranged from 0 to 144 months with patients surviving in the hormone refractory state (measured as time from first chemotherapy) ranging from 0 to 61 months with a hormone-refractory median survival of 14.5 months.

\section{ErbBI Immunohistochemistry}

TMAs from both groups were stained for expression of ErbB1 protein by means of a commercial kit utilizing a highly specific mouse monoclonal antibody (Clone H11, $\mathrm{DAKO}$, Carpenteria, CA). All staining procedures were carried out at room temperature, and all rinses were performed with DAKO wash buffer unless otherwise specified. TMAs were sectioned at $5 \mu$ thickness, dewaxed in three 2-min changes of xylene, rehydrated in graded alcohols for $2 \mathrm{~min}$ each, subjected to peroxidase block with 3\% hydrogen peroxide for $5 \mathrm{~min}$, rinsed, treated with proteinase $\mathrm{K}$ (DAKO) for $10 \mathrm{~min}$, rinsed, incubated with primary antibody or IgG1 isotype control for $60 \mathrm{~min}$, rinsed, incubated with linker reagent (dextran polymer conjugated with horseradish peroxidase and affinity-purified goat anti-mouse immunoglobulins, DAKO) for $30 \mathrm{~min}$, rinsed, reacted with $3,3^{\prime}$ diaminobenzidine in chromogen solution (DAKO) for $5 \mathrm{~min}$, rinsed in water, dehydrated in graded alcohols, incubated in three 2-min changes of xylene, and mounted in Permount under glass coverslips. Sections were examined by a pathologist (R.B.S.) in blinded fashion using a previously validated web-based TMA presentation tool, TMA Profiler (University of Michigan, Ann Arbor, MI) [20]. Isotype controls were uniformly negative (data not shown). ErbB1 staining was evaluated on a scale of 1 (negative), 2 (weak), 3 (moderate), and 4 (strong) staining intensity. Staining intensity for any given patient was defined as the median of the intensity score assigned to each core from that patient. In order to be scored, at least one of the cores had to contain tumor. ErbB1 $\leq 2$ and ErbB1 $>2$ expression groups were analyzed for association between ErbB1 protein expression (as assessed by staining intensity) and hormone-refractory status (by comparing hormone-naïve and hormone-refractory tumors) as well as a variety of other clinical parameters.

\section{Statistical Analysis}

All statistical inference was performed at a significance level of 0.05. EGFR staining was treated as a dichotomous variable (mean staining $\leq 2$ versus $>2$ ). Associations between continuous variables with ErbB1 staining were assessed using a $t$-test; those between categorical variables with ErbB1 staining were assessed using a chi-squared statistic. Across the tissue microarrays, multivariate logistic regression analysis was performed using ErbB1 staining as the response, and AIPC (yes/no), age (defined as age at diagnosis for the hormone-naïve group and age at death for the hormone-refractory group), and race (black/white) as the variables. In addition, time to event analyses were performed as well. For the nonmetastatic (i.e., hormone-naïve radical prostatectomy) TMAs, the endpoint of interest was biochemical failure (defined as elevation of PSA $>0.2 \mathrm{ng} / \mathrm{ml}$ ); for the hormone-refractory metastatic TMAs, the endpoint was death. The Kaplan-Meier method was used to estimate distributions of time to event. Proportional hazards regression models were used to model the time to event as a function of univariate and multiple covariates.

\section{RESULTS}

As reported previously [21-24], benign prostate glands adjacent to and distant from tumor tissues revealed strong ErbB1 staining, which was primarily localized to the basal layer compartment of the epithelium (Fig. 1A). In this compartment, ErbB1 protein expression was seen primarily in the form of membranous staining with weak intracytoplasmic staining, as reported previously [25].

ErbB1 expression in tumor cells was also predominantly localized to the cell membranes, though some granular cytoplasmic staining was also observed (Fig. 1B,D). ErbB1 expression in tumor tissues was strongly associated with hormone-refractory status (odds ratio $=6.67,95 \% \mathrm{CI}=(2.6,17.4), P<0.0001$ ), where the odds ratio compares the odds of high EGFR expression in a randomly chosen hormone-refractory tumor relative to a hormone-naïve tumor, adjusting for other covariates (Table I). In a proportional hazards model for biochemical failure of hormone-naïve clinically localized prostate cancer, ErbB1 overexpression was not a statistically significant covariate (Table II). Moreover, ErbB1 overexpression was not associated with tumor differentiation (Gleason score, $P=0.44$ ), positive margins $(P=0.53)$, seminal vesicle invasion $(P=0.69)$, extraprostatic extension $(P=0.10)$, or preoperative PSA $(P=0.18)$ in the hormone-naïve group (Table III).

In the hormone-refractory group, there was a trend toward poorer survival in individuals manifesting high $(>2)$ ErbB1 staining, as measured time from chemotherapy to death. However, this result was not 

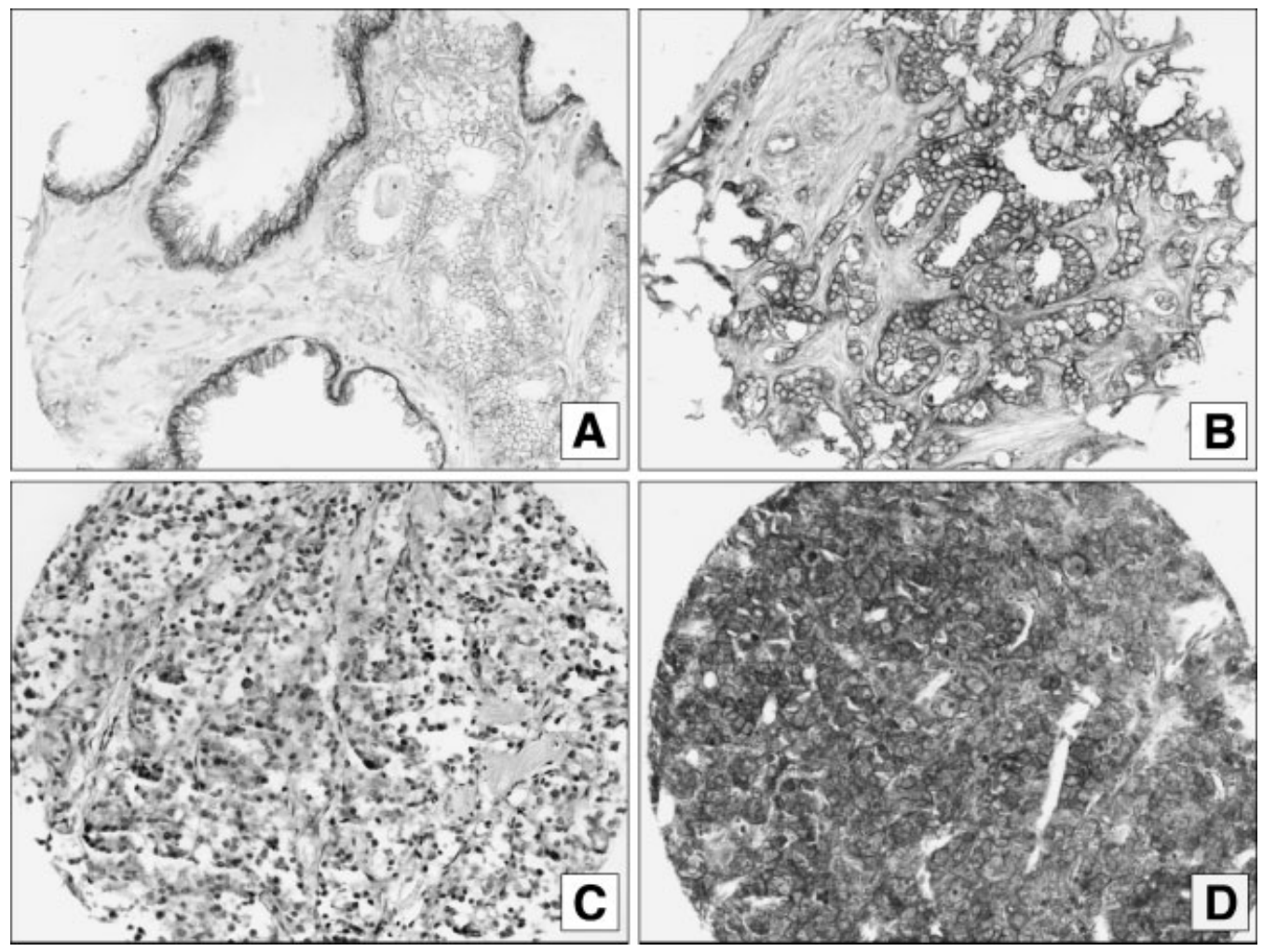

Fig. I. Examples of ErbBI staining patterns in benign prostate glands, hormone-naïve prostate cancer, and AIPC. Each image is representative of observations made on TMAs using the immunohistochemical staining protocol described in Materials and Methods. A: Normal prostate glands adjacent to a hormone-naïve tumor manifesting strong staining primarily localized to the basal compar tment. Tumor cells demonstrate weak (grade 2) membranous staining. B: Hormone-naïve prostate cancer demonstrating strong (grade 4) membranous ErbBl staining. C: Androgen-independent prostate cancer demonstrating lack of ErbBI expression (grade I). D: Androgen-independent prostate cancer demonstrating strong staining (grade 4), which in this tumor is membranous as well as cytoplasmic.

statistically significant (hazard ratio 1.32, 95\% CI (0.59, 2.96), $P=0.44)$. Mean ErbB1 staining scores exceeded the cutoff value of 2 for all metastatic sites, with the highest mean values observed in soft tissue, bone and lung (Table IV).

\section{DISCUSSION}

While a previous study had indicated that EGFR expression increases during the natural history of prostate cancer progression [7], this study could

TABLE I. Association Between ErbBI Expression and Androgen-Independent (Hormone-Refractory) Status

\begin{tabular}{lccc}
\hline Covariate & Odds ratio & $95 \%$ CI & $P$-value \\
\hline $\begin{array}{l}\text { Androgen-independent } \\
\text { prostate cancer }\end{array}$ & 6.67 & $(2.6,17.4)$ & 0.0001 \\
$\begin{array}{l}\text { Age } \\
\text { Race = black }\end{array}$ & 1.03 & $(0.98,1.09)$ & 0.21 \\
& 1.33 & $(0 / 49,3.59)$ & 0.57 \\
\hline
\end{tabular}

${ }^{a}$ Age is defined as age at diagnosis for the nonmetastatic (hormone-naive) TMAs and age at death for the metastatic (hormone-refractory) TMAs. not distinguish between androgen-dependent and androgen-independent tumors. More recently, a study appeared in which increased expression of ErbB1 was correlated with the transition from hormone-naïve to androgen-independent status [14]. Given the importance of signaling through ErbB1 to a wide variety of epithelial tumors via multiple potential mechanisms (proliferation, survival, migration, and invasion), we

TABLE II. Proportional Hazards Model for Biochemical Failure in Hormone-Naïve Clinically Localized Prostate Cancer

\begin{tabular}{lclc}
\hline Covariate & $\begin{array}{c}\text { Hazard } \\
\text { ratio }\end{array}$ & \multicolumn{1}{c}{$95 \%$ CI } & P-value \\
\hline ErbB1 staining $>2$ & 0.66 & $(0.22,1.93)$ & 0.45 \\
Extraprostatic extension & 1.50 & $(0.56,427)$ & 0.41 \\
Positive surgical margin & 4.16 & $(1.69,10.27)$ & 0.002 \\
Seminal vesicle invasion & 2.80 & $(0.86,9.11)$ & 0.09 \\
Ln (prediagnosis PSA +1$)^{\mathrm{a}}$ & 1.36 & $(1.10,1.66)$ & 0.004 \\
\hline
\end{tabular}

aPSA data were logarithmically transformed as shown because the distribution of PSA was skewed to the right and because some PSA values were equal to zero. 
TABLE III. Association Between ErbBI Expression and Clinical Parameters in HormoneNaïve Clinically Localized Prostate Cancers

\begin{tabular}{lccc}
\hline Clinical parameter & ErbB1 $>2$ & ErbB1 $\leq 2$ & $P$-value for association \\
\hline Age at diagnosis & 62.0 & 58.5 & 0.15 \\
Race = black & 36.3 & 32.2 & 0.30 \\
Gland weight & 50.3 & 50.7 & 0.90 \\
Gleason score & 6.8 & 6.6 & 0.44 \\
Maximum dimension & 1.37 & 1.41 & 0.23 \\
Seminal vesicle invasion & $4.5 \%$ & $6.6 \%$ & 0.69 \\
Positive surgical margin & $27.2 \%$ & $34.4 \%$ & 0.53 \\
Extraprostatic extension & $13.6 \%$ & $30.0 \%$ & 0.10 \\
Ln (prediagnosis PSA +1$)$ & 0.17 & 0.40 & 0.18 \\
\hline
\end{tabular}

wished to attempt confirmation of this observation. To accomplish this objective, we utilized TMAs containing two types of tumors: one containing clinically localized primary prostate cancers surgically removed prior to antiandrogen or radiation therapy, and another containing primary and metastatic tumors from individuals who died of hormone-refractory prostate cancer. Both TMAs were well characterized with respect to a variety of clinical parameters. Our results clearly confirmed the earlier findings of Di Lorenzo et al. [14], as the odds ratio for overexpression $(>2)$ of ErbB1 was 6.67 in androgen-independent relative to hormone-naïve prostate cancers (95\% CI (2.6, 17.4), $P=0.0001)$. Among hormone-naïve tumors, no correlations with any of the other clinical parameters could be detected in this sample, suggesting that increased expression of ErbB1 might be specifically connected to the development of androgen independence. However, our data do not directly demonstrate a causal role for ErbB1 in this process. For instance, it is possible that upregulation of ErbB1 is a straightforward consequence of transcriptional or post-transcriptional regulation related to androgen deprivation. Based on recent reports, we consider this to be unlikely. Torring et al. studied human androgen-sensitive CWR22 prostate cancer xenografts as a function of androgen depletion via castration, followed by QRT-PCR for multiple ErbB ligands and receptors [26]. While the ErbB1 ligands EREG and AREG were significantly increased in these xenografts as a function of androgen deprivation, there was actually a twofold decrease in ErbB1 expression within 2 weeks after castration, and this decrease was maintained until the end of the study (1 month after castration).

The fact that not all androgen-independent tumors displayed increased expression of ErbB1 (for example, see Fig. 1C) is likely a reflection of the fact that there are many pathways to androgen independence [27], which are reflected in substantial clinical and pathological heterogeneity of androgen-independent metastatic tumors, even within the same patient [17]. However, it is noteworthy that the mean ErbB1 staining intensity exceeded the cutoff point of 2 for all metastatic sites (Table IV).

Our results confirm those of another recent study, which was published after initiation of this work. Hernes et al. studied 106 symptomatic AIPC patients in whom prostatic biopsies were available before the start of androgen deprivation and after the development of AIPC. Expression of all four ErbB receptors was assessed by immunohistochemistry. It was found that only ErbB1 was significantly altered during the transition to androgen independence, being present in $69 \%$ of AIPC samples as opposed to $23 \%$ of androgen-naïve tumors [15]. That study contributes additional negative results to a conflicting literature regarding potential upregulation of ErbB2 in AIPC [28-30], a controversy not addressed in the present study.

Our results and those of Hernes et al. [15] differ somewhat from another study of ErbB1 and ErbB2 expression in prostate cancers before and after hormone therapy. In this smaller study of 49 patients, there was no significant increase in ErbB1 or ErbB2 staining with progression to androgen independence [16]. However, increased expression of ErbB1 or ErbB2 at hormone relapse was associated with significantly decreased survival in that study, consistent with

TABLE IV. ErbBI Staining Intensity Distributions by Site of Metastasis in Androgen-Independent Prostate Cancers

\begin{tabular}{lcccc}
\hline & No. of cores & Median & Mean & Variance \\
\hline Prostate & 39 & 3 & 2.46 & 0.99 \\
Soft tissue & 49 & 3 & 2.86 & 0.83 \\
Lymph node & 62 & 2 & 2.36 & 1.58 \\
Bone & 26 & 3 & 2.96 & 1.24 \\
Liver & 61 & 3 & 2.54 & 1.12 \\
Lung & 37 & 3 & 3.19 & 0.71 \\
\hline
\end{tabular}


the non-significant trend to decreased survival we observed in our ErbB1-overexpressing AIPC patients.

Our observations are consistent with a growing body of mechanistic evidence linking ErbB signaling and androgen independence, largely obtained utilizing cultured prostatic carcinoma cell lines. Thus, it has been shown that activation of ErbB signaling via ErbB2 overexpression can result in activation of the androgen receptor in an extracellular signal-responsive kinase (ERK)-dependent fashion [31]. More recently, it has been found that EGF triggers rapid association of Src and ErbB1 with the androgen receptor (AR) and estrogen receptor- $\beta(E R-\beta)$ in the prostate cancer cell line $\mathrm{LNCaP}$, in a process involving tyrosine phosphorylation of ER preassociated with AR, but not with Src or ErbB1 [32]. Remarkably, formation of this complex was necessary for EGF-induced tyrosine phosphorylation of ErbB1. Consistent with relevance of this pathway to multiple tumor types, increased Src family kinase (SFK) activity was also observed in tamoxifen-resistant MCF7 mammary carcinoma cells, pharmacological inhibition of SFKs led to decreased motility and invasiveness of the tamoxifen-resistant cells, and inhibition of ErbB RTKs markedly potentiated the effects of SFK inhibition [33]. While not explicitly linked to androgen/ estrogen responses, we have recently demonstrated a strong dependence of autocrine growth of normal human skin-derived keratinocytes upon SFK activation, and we have implicated SFKs in the shedding of AREG, a major autocrine ligand in keratinocytes [34]. Given the importance of autocrine and paracrine growth regulation in prostatic carcinoma [7,35], it will be of interest to examine the role of sex hormone receptors and SFK in the regulation of ErbB ligand shedding in pancreatic carcinoma cells.

Activation of ERK is a rapid downstream consequence of ErbB1 activation in a wide variety of epithelial cell types [36], suggesting that not only is AR is required for ErbB-dependent ERK activation, but also that ErbB activation activates AR transcriptional function via ERK. Consistent with this duality, hundreds of target genes were found to be stimulated by both EGF and dihydrotestosterone in an AR-dependent fashion in an SV40-immortalized prostate epithelial cell line [37]. Thus, while many important molecular details remain to be elucidated, these results suggest a multifaceted interaction between ErbB1 and AR in the determination of prostatic epithelial cell responses, and provide a framework for further mechanistic exploration of ErbB-dependent androgen resistance.

The expression patterns of ErbB1 in normal prostatic tissue versus prostatic carcinoma are of interest. In normal prostate and normal-appearing perilesional prostate tissue, we confirmed prior reports of staining confined predominantly to the basal layer compartment of the epithelium (Fig. 1A). The staining in the benign glands was primarily but not completely membranous. In contrast, patterns of ErbB1 expression in tumor tissue were variable, with some tumors demonstrating only membranous staining while others demonstrated membranous as well as cytoplasmic staining (Fig. 1D). These results emphasize the importance of regulated ErbB1 expression and localization for normal prostatic function, and emphasize a need for better understanding of the downstream pathways coupling ErbB1 to cellular responses in normal versus malignant prostate tissue.

The correlation that we and others have observed between increased ErbB1 expression and progression to androgen independence suggests that EGFR-targeted drugs could be of therapeutic relevance in the management of advanced prostate cancer. However, the marked heterogeneity of ErbB1 expression that we have observed in this study indicates that it will be important to target such therapy to those individuals who are overexpressing ErbB1. Moreover, for prostate cancer as well as other tumors in which aberrant ErbB signaling has been implicated, including non-small-cell lung cancer [38], glioblastoma multiforme [39], and breast cancer [40], patient-by-patient characterization of post-receptor signaling alterations capable of overriding the therapeutic effects of ErbB1 RTK inhibition, such as loss of PTEN [39], will likely be necessary to identify appropriate candidates for therapeutic inhibition of ErbB1 activity in prostatic carcinoma patients.

\section{ACKNOWLEDGMENTS}

This research was supported by an award (P50CA69568) from the National Cancer Institute (JTE, DG, RS), by the Ann Arbor Veterans Affairs Hospital (JTE), and by the Babcock Memorial Trust (JTE).

\section{REFERENCES}

1. Tannock IF, Osoba D, Stockler MR, Ernst DS, Neville AJ, Moore MJ, Armitage GR, Wilson JJ, Venner PM, Coppin CM, Murphy KC. Chemotherapy with mitoxantrone plus prednisone or prednisone alone for symptomatic hormone-resistant prostate cancer: A Canadian randomized trial with palliative end points. J Clin Oncol 1996;14(6):1756-1764.

2. Russell PJ, Bennett S, Stricker P, Tannock IF, Osoba D, Stockler MR, Ernst DS, Neville AJ, Moore MJ, Armitage GR, Wilson JJ, Venner PM, Coppin CM, Murphy KC. Growth factor involvement in progression of prostate cancer. Clin Chem 1998;44(4): 705-723.

3. Tillotson JK, Rose DP. Endogenous secretion of epidermal growth factor peptides stimulates growth of DU145 prostate cancer cells. Cancer Lett 1991;60(2):109-112.

4. Tillotson JK, Rose DP. Density-dependent regulation of epidermal growth factor receptor expression in DU 145 human prostate cancer cells. Prostate 1991;19(1):53-61. 
5. Traish AM, Wotiz HH. Prostatic epidermal growth factor receptors and their regulation by androgens. Endocrinology 1987;121(4):1461-1467.

6. Hofer DR, Sherwood ER, Bromberg WD, Mendelsohn J, Lee C, Kozlowski JM. Autonomous growth of androgen-independent human prostatic carcinoma cells: Role of transforming growth factor alpha. Cancer Res 1991;51(11):2780-2785.

7. Scher HI, Sarkis A, Reuter V, Cohen D, Netto G, Petrylak D, Lianes P, Fuks Z, Mendelsohn J, Cordon-Cardo C. Changing pattern of expression of the epidermal growth factor receptor and transforming growth factor alpha in the progression of prostatic neoplasms. Clin Cancer Res 1995;1(5):545550.

8. Klapper LN, Kirschbaum MH, Sela M, Yarden Y. Biochemical and clinical implications of the ErbB/HER signaling network of growth factor receptors. Adv Cancer Res 2000;77:25-79.

9. Olayioye MA, Neve RM, Lane HA, Hynes NE. The ErbB signaling network: Receptor heterodimerization in development and cancer. EMBO J 2000;19(13):3159-3167.

10. Hackel PO, Zwick E, Prenzel N, Ullrich A. Epidermal growth factor receptors: Critical mediators of multiple receptor pathways. Curr Opin Cell Biol 1999;11(2):184-189.

11. Herbst RS, Onn A, Mendelsohn J. The role of growth factor signaling in malignancy. Cancer Treat Res 2003;115:19-72.

12. Mendelsohn J. The epidermal growth factor receptor as a target for cancer therapy. Endocr Relat Cancer 2001;8(1):3-9.

13. Bostwick DG, Qian J, Maihle NJ. Amphiregulin expression in prostatic intraepithelialneoplasia and adenocarcinoma: A study of 93 cases. Prostate 2004;58(2):164-168.

14. Di Lorenzo G, Tortora G, D'Armiento FP, De Rosa G, Staibano S, Autorino R, D'Armiento M, De Laurentiis M, De Placido S, Catalano G, Bianco AR, Ciardiello F. Expression of epidermal growth factor receptor correlates with disease relapse andprogression to androgen-independence in human prostate cancer. Clin Cancer Res 2002;8(11):3438-3444.

15. Hernes E, Fossa SD, Berner A, Otnes B, Nesland JM. Expression of the epidermalgrowth factor receptor family in prostate carcinoma before and during androgen-independence. Br J Cancer 2004;90(2):449-454.

16. Bartlett JM, Brawley D, Grigor K, Munro AF, Dunne B, Edwards J. Type I receptor tyrosine kinases are associated with hormone escape in prostate cancer. J Pathol 2005;205(4):522-529.

17. Shah RB, Mehra R, Chinnaiyan AM, Shen R, Ghosh D, Zhou M, Macvicar GR, Varambally S, Harwood J, Bismar TA, Kim R, Rubin MA, Pienta KJ. Androgen-independent prostate cancer is a heterogeneous group of diseases: Lessons from a rapid autopsy program. Cancer Res 2004;64(24):9209-9216.

18. Rubin MA, Dunn R, Strawderman M, Pienta KJ. Tissue microarray sampling strategy for prostate cancer biomarker analysis. Am J Surg Pathol 2002;26(3):312-319.

19. Kononen J, Bubendorf L, Kallioniemi A, Barlund M, Schraml P, Leighton S, Torhorst J, Mihatsch MJ, Sauter G, Kallioniemi OP. Tissue microarrays for high-throughput molecular profiling of tumor specimens. Nat Med 1998;4(7):844-847.

20. Manley S, Mucci NR, De Marzo AM, Rubin MA. Relational database structure to manage high-density tissue microarray data and images for pathology studies focusing on clinical outcome: The prostate specialized program of research excellence model. Am J Pathol 2001;159(3):837-843.

21. De Miguel P, Royuela M, Bethencourt R, Ruiz A, Fraile B, Paniagua R. Immunohistochemical comparative analysis of transforming growth factor alpha, epidermal growth factor, and epidermal growth factor receptor in normal, hyperplastic and neoplastic human prostates. Cytokine 1999;11(9):722727.

22. Leav I, McNeal JE, Ziar J, Alroy J. The localization of transforming growth factor alpha and epidermal growth factor receptor in stromal and epithelial compartments of developing human prostate and hyperplastic, dysplastic, and carcinomatous lesions. Hum Pathol 1998;29(7):668-675.

23. Myers RB, Kudlow JE, Grizzle WE. Expression of transforming growth factor-alpha, epidermal growth factor and the epidermal growth factor receptor in adenocarcinoma of the prostate and benign prostatic hyperplasia. Mod Pathol 1993;6(6):733-737.

24. Maygarden SJ, Strom S, Ware JL. Localization of epidermal growth factor receptor by immunohistochemical methods in human prostatic carcinoma, prostatic intraepithelial neoplasia, and benign hyperplasia. Arch Pathol Lab Med 1992;116(3):269273.

25. Damjanov I, Mildner B, Knowles BB. Immunohistochemical localization of the epidermal growth factor receptor in normal human tissues. Lab Invest 1986;55(5):588-592.

26. Torring N, Hansen FD, Sorensen BS, Orntoft TF, Nexo E. Increase in amphiregulin and epiregulin in prostate cancer xenograft after androgen deprivation-impact of specific HER1 inhibition. Prostate 2005;64(1):1-8.

27. Culig Z, Steiner H, Bartsch G, Hobisch A. Mechanisms of endocrine therapy-responsive and -unresponsive prostate tumours. Endocr Relat Cancer 2005;12(2):229-244.

28. Di Lorenzo G, Autorino R, De Laurentiis M, Cindolo L, D'Armiento M, Bianco AR, De Placido S. HER-2/neu receptor in prostate cancer development and progression to androgen independence. Tumori 2004;90(2):163-170.

29. Kuhn EJ, Kurnot RA, Sesterhenn IA, Chang EH, Moul JW, deFazio A, Chiew YE, Sini RL, Janes PW, Sutherland RL. Expression of the c-erbB-2 (HER-2/neu) oncoprotein in human prostatic carcinoma. J Urol 1993;150(5 Pt 1):1427-1433.

30. Myers RB, Srivastava S, Oelschlager DK, Grizzle WE. Expression of p160erbB-3 and p185erbB-2 in prostatic intraepithelial neoplasia and prostatic adenocarcinoma. J Natl Cancer Inst 1994;86(15):1140-1145.

31. Yeh S, Lin HK, Kang HY, Thin TH, Lin MF, Chang C. From HER2/Neu signal cascade to androgen receptor and its coactivators: A novel pathway by induction of androgen target genes through MAP kinase in prostate cancer cells. Proc Natl Acad Sci USA 1999;96(10):5458-5463.

32. Migliaccio A, Di Domenico M, Castoria G, Nanayakkara M, Lombardi M, de Falco A, Bilancio A, Varricchio L, Ciociola A, Auricchio F. Steroid receptor regulation of epidermal growth factor signaling through Src in breast and prostate cancer cells: Steroid antagonist action. Cancer Res 2005;65(22):1058510593.

33. Hiscox S, Morgan L, Green TP, Barrow D, Gee J, Nicholson RI. Elevated Src activity promotes cellular invasion and motility in tamoxifen resistant breast cancer cells. Breast Cancer Res Treat 2005:94(3):1-12.

34. Kansra S, Stoll SW, Johnson JL, Elder JT. Src family kinase inhibitors block amphiregulin-mediated autocrine ErbB signaling in normal human keratinocytes. Mol Pharmacol 2005;67(4): 1145-1157.

35. Mimeault M, Brand RE, Sasson AA, Batra SK. Recent advances on the molecular mechanisms involved in pancreatic cancer progression and therapies. Pancreas 2005;31(4):301-316. 
36. Kansra S, Stoll SW, Johnson JL, Elder JT. Autocrine extracellular signal-regulated kinase (ERK) activation in normal human keratinocytes: Metalloproteinase-mediated release of amphiregulin triggers signaling from ErbB1 to ERK. Mol Biol Cell 2004;15(9):4299-4309.

37. York TP, Plymate SR, Nelson PS, Eaves LJ, Webb HD, Ware JL. cDNA microarray analysis identifies genes induced in common by peptide growth factors and androgen in human prostate epithelial cells. Mol Carcinog 2005;44(4):242-251.

38. Bell DW, Lynch TJ, Haserlat SM, Harris PL, Okimoto RA, Brannigan BW, Sgroi DC, Muir B, Riemenschneider MJ, Iacona RB, Krebs AD, Johnson DH, Giaccone G, Herbst RS, Manegold C, Fukuoka M, Kris MG, Baselga J, Ochs JS, Haber DA. Epidermal growth factor receptor mutations and gene ampli- fication in non-small-cell lung cancer: Molecular analysis of the IDEAL/INTACT gefitinib trials. J Clin Oncol 2005; 23(31): 8081-8092.

39. Mellinghoff IK, Wang MY, Vivanco I, Haas-Kogan DA, Zhu S, Dia EQ, Lu KV, Yoshimoto K, Huang JH, Chute DJ, Riggs BL, Horvath S, Liau LM, Cavenee WK, Rao PN, Beroukhim R, Peck TC, Lee JC, Sellers WR, Stokoe D, Prados M, Cloughesy TF, Sawyers CL, Mischel PS. Molecular determinants of the response of glioblastomas to EGFR kinase inhibitors. N Engl J Med 2005;353(19):2012-2024.

40. She QB, Solit DB, Ye Q, O'Reilly KE, Lobo J, Rosen N. The BAD protein integrates survival signaling by EGFR/MAPK and PI3K/Akt kinase pathways in PTEN-deficient tumor cells. Cancer Cell 2005;8(4):287-297. 